\title{
Organization of Intralaminar and Translaminar Neuronal Connectivity in the Superficial Spinal Dorsal Horn
}

\author{
Go Kato, ${ }^{1}$ Yasuhiko Kawasaki, ${ }^{2}$ Kohei Koga, ${ }^{3}$ Daisuke Uta, ${ }^{3}$ Masafumi Kosugi, ${ }^{1}$ Toshiharu Yasaka, ${ }^{4}$ \\ Megumu Yoshimura, ${ }^{3} \mathrm{Ru}$-Rong Ji, ${ }^{2}$ and Andrew M. Strassman ${ }^{1}$ \\ Department of Anesthesia, ${ }^{1}$ Beth Israel Deaconess Medical Center and ${ }^{2}$ Brigham and Women's Hospital, Harvard Medical School, Boston, Massachusetts \\ 02215, ${ }^{3}$ Department of Integrative Physiology, Kyushu University, School of Medicine, Fukuoka 812-8582, Japan, and ${ }^{4}$ Spinal Cord Group, Institute of \\ Biomedical and Life Sciences, University of Glasgow, Glasgow G12 8QQ, United Kingdom
}

The spinal dorsal horn exhibits a high degree of intrinsic connectivity that is critical to its role in the processing of nociceptive information. To examine the spatial organization of this intrinsic connectivity, we used laser-scanning photostimulation in parasagittal and transverse slices of lumbar spinal cord to stimulate presynaptic neurons by glutamate uncaging, and mapped the location of sites that provide excitatory and inhibitory synaptic input to neurons of the superficial laminae. Excitatory interneuronal connectivity within lamina II exhibited a pronounced sagittal orientation, in keeping with the somatotopic organization present in the pattern of primary afferent projections. Excitatory inputs to all classes of lamina II neurons arose from a wider rostrocaudal area than inhibitory inputs, whereas both excitatory and inhibitory input zones were restricted mediolaterally. Lamina I-II neurons exhibited cell type-specific patterns in the laminar distribution of their excitatory inputs that were related to their dorsoventral dendritic expanse. All cell types received excitatory input predominantly from positions ventral to that of their soma, but in lamina I neurons and lamina II vertical cells this ventral displacement of the excitatory input zone was greater than in the other cell types, resulting in a more pronounced translaminar input pattern. A previously unknown excitatory input to the superficial dorsal horn from lamina III-IV was identified in a subset of the vertical cell population. These results reveal a specific three-dimensional organization in the local patterns of excitatory and inhibitory connectivity that has implications for the processing of information related to both somatotopy and sensory modality.

\section{Introduction}

The spinal dorsal horn is a major termination site of primary afferent fibers carrying sensory information from the periphery and contains projection neurons that transmit this sensory information to higher levels of the CNS (Willis and Coggeshall, 2004). The primary afferent terminations exhibit a specific laminar organization, such that mechanosensitive A- $\beta$ fibers, carrying tactile information, terminate primarily in the deep laminae (III-V), whereas the superficial laminae (I-II) receive projections primarily from the smaller A- $\delta$ and C fibers, many of which are nociceptive (Light, 1992). In addition to receiving direct primary afferent input, dorsal horn neurons participate in a high degree of local interneuronal connectivity that can also influence their sensory response properties, as shown by the large amount of polysynaptic input evoked by dorsal root stimulation (Yoshimura and Nishi, 1992, 1995; Torsney and MacDermott, 2006; Yasaka et al., 2007). Such polysynaptic input would be expected to be weaker than direct input but also show greater modifiability as a result of

Received Dec. 29, 2008; revised Feb. 12, 2009; accepted Feb. 19, 2009.

This work was supported by National Institutes of Health Grants R21 NS047371 and R01 NS057454. We thank Daniel Robichaud III for LabView programming, and Elliot Strassman for assistance with data analysis.

This article is freely available online through the J Neurosci Open Choice option.

Correspondence should be addressed to Go Kato, Department of Orthopaedic Surgery, Graduate School of Medical Sciences, Kyushu University, 3-1-1 Maidashi Higashi-ku, Fukuoka 812-8582, Japan. E-mail: gkato@ortho. med.kyushu-u.ac.jp.

DOI:10.1523/JNEUROSCI.6175-08.2009

Copyright $\odot 2009$ Society for Neuroscience $\quad$ 0270-6474/09/295088-12\$15.00/0 changes in synaptic strength. A small percentage of superficial dorsal horn neurons normally receive polysynaptic A- $\beta$ input, but this percentage is greatly increased under conditions of central disinhibition (Torsney and MacDermott, 2006) and in animal models of inflammatory or neuropathic pain hypersensitivity (Baba et al., 1999; Nakatsuka et al., 1999; Okamoto et al., 2001; Kohno et al., 2003). It has been hypothesized that this polysynaptic A- $\beta$ input is mediated by an as-yet-unidentified interneuronal connection from the deep to the superficial laminae (Torsney and MacDermott, 2006).

Important advances in the study of intrinsic dorsal horn organization have come from the application of novel methods of genetically based neuronal tracing (Braz et al., 2005) and dual microelectrode recording and stimulation techniques ( $\mathrm{Lu}$ and Perl, 2003, 2005; Santos et al., 2007). The paired recording method has been successful in identifying cell type-specific patterns of inhibitory and excitatory connectivity (Lu and Perl, 2003, 2005) but is generally not as well suited for revealing patterns of spatial organization. A high degree of spatial organization has been found in the pattern of local connectivity in laminated regions such as primary visual and somatosensory cortex, but it is not clear to what degree such organization is exhibited by the intrinsic circuitry of the dorsal horn. One method that is well suited to examining spatial organization of local connectivity is laser-scanning photostimulation (LSPS), which allows the mapping of local sites that are monosynaptically connected to a single postsynaptic neuron (Callaway and Katz, 1993; Katz and Dalva, 
1994). We previously used this method to map the location of local excitatory and inhibitory inputs to one major class of lamina II inhibitory interneuron, the islet cell (Kato et al., 2007). In the present study, this method is extended to other cell types in lamina I and II. The results reveal a specific three-dimensional organization of local excitatory and inhibitory connectivity within the superficial laminae and also identify an input from lamina III-IV that could serve to relay polysynaptic A- $\beta$ input to nociceptive superficial dorsal horn neurons.

\section{Materials and Methods}

Methods for spinal cord slice preparation, whole-cell recording, and laser-scanning photostimulation were as described by Kato et al. (2007), except for the following changes: (1) recordings were obtained blind at depths of $50-100 \mu \mathrm{m}$ below the surface of the slice, rather than by infrared-differential interference contrast (IR-DIC)-guided visualization of neurons at more superficial depths, and (2) both transverse and parasagittal slices were used.

Overview of LSPS method, including monosynaptic nature of excitation, and determination of spatial resolution. Laser-scanning photostimulation is a method for mapping the locations of local neurons that are synaptically connected to a single identified neuron and for identifying those connections as excitatory or inhibitory (Callaway and Katz, 1993; Katz and Dalva, 1994; Callaway and Yuste, 2002). A whole-cell patch-clamp recording is obtained from a candidate postsynaptic neuron in a slice preparation that is bathed in a solution containing caged glutamate while the beam from a UV laser is systematically scanned across the slice. At each scanning site, the laser produces focal uncaging of the caged glutamate, which in turn evokes firing of neurons whose cell bodies are near that site. If one of these neurons is presynaptic to the recorded neuron, then synaptic responses are evoked in the recorded neuron.

Two methodological points are that the glutamate directly activates only cell bodies or dendrites, not axons of passage, and that the photostimulus only reveals monosynaptic, not polysynaptic, connections. Both of these conclusions are based on the finding, demonstrated by other investigators as well as in our previous study, that action potentials can only be evoked when photostimulating at sites on the soma or dendrites; stimulating at other sites can evoke only subthreshold synaptic potentials, not action potentials (Katz and Dalva, 1994; Sawatari and Callaway, 2000; Kato et al., 2007). This is evidence that synaptically evoked responses do not reach threshold, and therefore polysynaptic transmission cannot occur. One caveat regarding this observation is that the excitability of the recorded neuron might be lower than that of other neurons in the slice, as a result of the intracellular perfusion from the patch pipette. Therefore, the possibility cannot be excluded that exceptionally strong polysynaptic inputs might also be evoked by the photostimulus. However, the fact that evidence for this has never been observed, in any studies with photostimulation, suggests that it is not common. Apparently, this is an indication that, in the superficial dorsal horn, as well as in other regions that have been studied with this method, threshold for action potential initiation requires summation of synaptic input from a number of neurons, and the maximum number of presynaptic neurons activated by the photostimulus is typically not sufficient to produce this degree of summation. This presumably is a reflection of the spatial and temporal properties of the photostimulus, as well as the density of local synaptic connectivity. We have no estimate of the number of neurons activated by the photostimulus, but the laser beam used in the present study was $10 \mu \mathrm{m}$ in diameter, and it has been estimated that the UV light used for uncaging may penetrate up to $100 \mu \mathrm{m}$ into the tissue. The effective size of the stimulus is the total area surrounding the stimulus in which neurons can be depolarized to threshold. This was determined in our previous study by mapping the location of stimulation sites that evoke action potentials, for a sample of neurons in different dorsal horn laminae (Kato et al., 2007). In the present study, we have compared such action potential maps for lamina II islet cells and nonislet cells, as way to partly address the question of whether the sensitivity and resolution of the synaptic mapping might differ for excitatory versus inhibitory inputs (supplemental Fig. 2 and accompanying text, available at www. jneurosci.org as supplemental material).

Slice preparation. All experimental procedures involving the use of animals were approved by the Institutional Animal Care and Use Committee of the Beth Israel Deaconess Medical Center. Parasagittal or transverse slices of $250 \mu \mathrm{m}$ thickness (see Fig. $1 A-C$ ) were prepared from the enlargement of the lumbar spinal cord of rats at the postnatal age of 35-65 d (mostly 35-50 d), using methods similar to those described previously (Yoshimura and Jessell, 1989; Bentley and Gent, 1994). Slices were transferred to a recording chamber, placed on the stage of an upright microscope equipped with IR-DIC, and perfused continuously at a flow rate of $10-20 \mathrm{ml} / \mathrm{min}$ with Krebs' solution equilibrated with $95 \% \mathrm{O}_{2}$ and $5 \% \mathrm{CO}_{2}$. The Krebs' solution was maintained at room temperature for $1 \mathrm{~h}$ and was then warmed to $36 \pm 1^{\circ} \mathrm{C}$ before recording. The Krebs' solution contained the following (in $\mathrm{mM}$ ): $117 \mathrm{NaCl}, 3.6 \mathrm{KCl}, 2.5 \mathrm{CaCl}_{2}$, $1.2 \mathrm{MgCl}_{2}, 1.2 \mathrm{NaH}_{2} \mathrm{PO}_{4}, 25 \mathrm{NaHCO}_{3}$, and 11 glucose. The circulating Krebs' solution contained MNI-caged L-glutamate (4-methoxy-7nitroindolinyl-caged L-glutamate) (Tocris) at a concentration of $100 \mu \mathrm{M}$.

Recording and cell class identification. Laminar regions were identified under a $10 \times$ water-immersion objective (see Fig. $1 B, C$ ). After dye filling, individual neurons were visualized with a $40 \times$ water-immersion objective under epifluorescent illumination. The patch pipettes (6-8 M $\Omega$ ) were filled with a solution containing the following (in $\mathrm{mM}$ ): 136 K-gluconate, $5 \mathrm{KCl}, 0.5 \mathrm{CaCl}_{2}, 2 \mathrm{MgCl}_{2}, 5 \mathrm{EGTA}, 5 \mathrm{HEPES}$, and $5 \mathrm{Mg}$ ATP for recording EPSCs in the voltage-clamp mode; $110 \mathrm{Cs}_{2} \mathrm{SO}_{4}, 5$ TEA-Cl, $0.5 \mathrm{CaCl}_{2}, 2 \mathrm{MgCl}_{2}, 5$ EGTA, 5 HEPES, and $5 \mathrm{Mg}$-ATP for recording inhibitory outward currents in the voltage-clamp mode. Whole-cell recordings were obtained from neurons at a depth of 50-100 $\mu \mathrm{m}$ in the slice. A fluorescent dye (Alexa 555 hydrazide, Tris salt; $40 \mu \mathrm{M}$; Invitrogen) was added to the pipette solution to visualize the neuron during the recording periods. Neurobiotin $(0.1 \%$; Vector) was also added for additional anatomical examination after histological processing. Mammalian lamina II neurons were classified into four major groups (islet, central, radial, and vertical cells) as visualized by fluorescent dye or Neurobiotin staining in parasagittal sections (Grudt and Perl, 2002; Yasaka et al., 2007). The islet and central cells in this classification scheme correspond to the large and small islet cells, respectively, in the terminology used by some other investigators (Todd and McKenzie, 1989; Eckert et al., 2003) (see additional discussion of these terminologies in text accompanying supplemental Fig. 2, available at www.jneurosci. org as supplemental material).

Signals were amplified and filtered at $4 \mathrm{kHz}$ (Multiclamp 700B; Molecular Devices) and sampled at $10 \mathrm{kHz}$. The data were digitized with an analog-to-digital converter (Digidata 1322A; Molecular Devices) and stored on a personal computer using a data acquisition program (Clampex, version 9.2; Molecular Devices). Recording of action potentials was done under current clamp. Excitatory and inhibitory synaptic responses were recorded under voltage clamp in separate experiments, at different holding potentials. Excitatory (inward) currents were recorded at a holding potential of $-70 \mathrm{mV}$. This potential was close to the reversal potential for $\mathrm{GABA}_{\mathrm{A}^{-}}$and glycine-mediated responses, so IPSCs were minimized and usually undetectable. Inhibitory (outward) currents were recorded at a holding potential of $0 \mathrm{mV}$. At this potential, inward EPSCs mediated by glutamate receptors were minimized and usually undetectable; all outward currents are abolished by strychnine and bicuculline, showing that there is no detectable contamination of outward currents by reversed EPSCs, under these recording conditions (Yasaka et al., 2007).

As explained by Kato et al. (2007), recording of action potentials was done in the initial experiments with this technique to determine the spatial resolution of the synaptic mapping in the spinal dorsal horn, because the effective size of the stimulus corresponds to the total area surrounding the stimulus in which neurons are depolarized to threshold. The results of such action potential mapping experiments were reported for neurons in different dorsal horn laminae by Kato et al. (2007). We have now also compared such action potential maps for lamina II islet cells and nonislet cells, as way to partly address the question of whether the sensitivity and resolution of the synaptic mapping might differ for excitatory versus inhibitory inputs (supplemental Fig. 2, available at www. jneurosci.org as supplemental material). The figure shows no difference 
in the sensitivity to photostimulation but does show that islet cells can be activated over a greater rostrocaudal distance, paralleling their greater rostrocaudal dendritic extent.

Laser-scanning photostimulation. The laser and associated optical apparatus for delivering the beam to the specimen were obtained from Prairie Technologies. The light source was a Coherent Enterprise watercooled continuous argon ion laser (ENT-653 or ENT-II-653) with a total output of $4 \mathrm{~W}$ and a UV output of $60 \mathrm{~mW}$. The laser beam was coupled to a fiber optic that led to a single-path epi photolysis head, which was incorporated into an Olympus BX51W1 fixed-stage microscope. The $40 \times$ water-immersion objective (numerical aperture, 0.8 ) of the microscope was used both for viewing the tissue and for transmitting and focusing the UV beam to the tissue for photostimulation. The UV power at the specimen was $\sim 5 \mathrm{~mW}$. The epi photolysis head has a motorized $x-y$ positioning plate for positioning the fiber optic input within the microscope field of the $40 \times$ objective. Scanning was done by mounting the microscope on an $x-y$ motorized stage (Scientifica) while the tissue was mounted independently on a fixed platform. The stimulus site was changed by moving the entire microscope, so the laser beam was always centered within the microscope objective and the microscope field, and the optical properties of the stimulus were identical for all stimulus sites. Stage movement was controlled by custom software written in LabView 7.1 (National Instruments). The parameters for laser intensity, flash duration, and caged glutamate concentration ( $5 \mathrm{~mW}, 100 \mu \mathrm{M}$, and $3 \mathrm{~ms}$ ) were unchanged from our previous study, so that the analysis of spatial and temporal characteristics of photostimulation-evoked responses from that study is applicable to the present data. In mapping experiments, photostimulation was delivered at $0.8 \mathrm{~Hz}$, in grids with $25 \mu \mathrm{m}$ between stimulation sites. In parasagittal slices, the stimulation grid had dorsoventral by rostrocaudal dimensions of $375 \times 1175 \mu \mathrm{m}$ (768 sites). In transverse slices, it had dorsoventral by mediolateral dimensions of $475 \times 975 \mu \mathrm{m}$ (800 sites) (see Fig. 1 B,C). These dimensions were sufficient to cover laminae I-IV. Our initial data sample in parasagittal slices, including each of the major cell types, used a larger dorsoventral dimension of $475 \mu \mathrm{m}$, but input zones were never observed in the ventralmost $150 \mu \mathrm{m}$, so this dimension was reduced to $375 \mu \mathrm{m}$ in subsequent experiments. The sites were stimulated in a sequence generated by a nonneighbor algorithm, to avoid receptor desensitization and local depletion of the caged compound.

Histology. After termination of the electrophysiological recordings, the spinal cord slice was immersed for $1 \mathrm{~h}$ in $4 \%$ paraformaldehyde in $0.1 \mathrm{M}$ phosphate buffer, $\mathrm{pH} 7.4$, at $4^{\circ} \mathrm{C}$, and then overnight in $15 \%$ sucrose in $0.1 \mathrm{~m}$ PBS. Sectioning of the slice was not performed to preserve the dendrites of the Neurobiotin-stained neuron intact. The slice was blocked with $0.5 \%$ bovine serum albumin in $0.3 \%$ Triton X-100 for $1 \mathrm{~h}$ at room temperature and incubated overnight at $4^{\circ} \mathrm{C}$ with streptavidin conjugated to Alexa Fluor 488 (diluted 1:500; Invitrogen). The slice was washed in PBS, mounted on slides, and viewed with a Nikon fluorescence microscope, and the images were captured with a high-resolution CCD Spot camera (Diagnostic Instruments).

Data analysis. In each stimulus trial, electrophysiological data were acquired for $100 \mathrm{~ms}$ before and $300 \mathrm{~ms}$ after the photostimulus. Synaptic events as well as direct responses were detected with the aid of MiniAnalysis software (Synaptosoft) and confirmed by visual inspection. Peak amplitude and time of onset were determined for each event. Response amplitude for each trial (stimulation site) was measured as the sum of the peak amplitudes of all of the events whose onset occurred within the defined response time window. The response time window was $0-5 \mathrm{~ms}$ (relative to stimulus onset) for direct responses and 6-106 ms for synaptic responses (Kato et al., 2007) (see Fig. 1D). Spontaneous activity was measured in the same way, during the $100 \mathrm{~ms}$ prestimulus interval. Spontaneous activity was calculated as a moving average of the prestimulus activity over 100 stimulus trials. This moving average of spontaneous activity was then subtracted from the response calculated for each stimulus trial.

Construction of individual and averaged maps of synaptic input zones. A color-coded contour map was made for each neuron showing the amplitude of the synaptic response evoked from each stimulation site in the photostimulation scanning grid. These are referred to as maps of synaptic input zones. Averaging of individual maps was performed as described previously (Kato et al., 2007). First, response amplitudes for each neuron were normalized relative to the mean amplitude of the spontaneous events recorded in that neuron [similar to Dantzker and Callaway (2000)]. Responses were divided by a normalization factor that was calculated as the mean spontaneous EPSC amplitude in that neuron divided by the mean spontaneous EPSC amplitude in all of the neurons $(\sim 15.3$ $\mathrm{pA})$. For construction of averaged maps, individual maps were aligned dorsoventrally either by soma location (see Fig. 2) or by the borders of lamina II (see Figs. 3-5). For alignment by laminar borders, because there is some variation between slices in the dorsoventral width of lamina II, the dorsoventral dimension of each map was normalized before averaging to make lamina II a standard width $(150 \mu \mathrm{m})$. For both alignment methods, a method was used to straighten the individual maps before averaging, which involved shifting all of the dorsoventral coordinates at each rostrocaudal position so that the dorsoventral coordinate of the lamina II-III border had the same value across the entire map.

Rostrocaudal and dorsoventral weighted mean and half-width calculations. For each neuron, separate plots were made of the amplitude of the evoked responses along the rostrocaudal and dorsoventral axes (similar to the averaged plots in Fig. 2 E, F, respectively). The value at each coordinate in the plots represents the sum of the response amplitudes of all the sites with that coordinate (summing across all dorsoventral positions for each point in the rostrocaudal plot, and all rostrocaudal positions for each point in the dorsoventral plot). For the rostrocaudal plots, the values at negative and positive coordinates (positions at equal distances rostral and caudal to the soma) were combined to make a plot of rostrocaudal distance from the soma. A calculation was then made of the weighted mean of each plot, as an estimate of the position of the center of the distribution. A calculation was also made of the total distance encompassing all positions that had greater than one-half of the maximum amplitude ("half-width"), as a measure of the width of the distribution.

Statistics. Unpaired $t$ tests or repeated-measures ANOVA followed by Bonferroni-Dunn post hoc tests were used to compare the population data. All averaged data are represented as mean \pm SEM. Asterisks indicate signif-

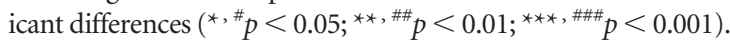

\section{Results}

Whole-cell recordings were obtained from neurons in the superficial dorsal horn (laminae I-II) in both parasagittal and transverse slices made from the enlargement of the lumbar spinal cord (Fig. $1 A-C$ ). Resting membrane potentials were $-61.1 \pm 1.4 \mathrm{mV}$ for lamina I and $-65.0 \pm 0.5 \mathrm{mV}$ for lamina II neurons. Input resistances were $623 \pm 57 \mathrm{M} \Omega$ for lamina I and $532 \pm 22 \mathrm{M} \Omega$ for lamina II neurons (central, $519 \pm 72$ $\mathrm{M} \Omega$; radial, $592 \pm 42 \mathrm{M} \Omega$; islet, $465 \pm 51 \mathrm{M} \Omega$; vertical, $466 \pm$ $39 \mathrm{M} \Omega$ ). Scanning grids for photostimulation covered the superficial laminae (I-II) and extended $\sim 200 \mu \mathrm{m}$ into the deep dorsal horn. Our previous study described the spatial resolution of the photostimulation method in the dorsal horn, as determined by action potential mapping, and established the latency criterion for distinguishing direct versus synaptically evoked responses, as determined by TTX sensitivity (Kato et al., 2007). To illustrate the validity of this latency criterion, Figure $1 \mathrm{D} 1$ shows responses evoked in one lamina II radial cell from photostimulation at the four sites $(a-d)$ indicated in the maps in Figure 1D2. Direct responses (latency, 0-5 ms) were only evoked from sites on the soma and proximal dendrites (Fig. 1 D2, top left) and were TTX-resistant (bottom left). Synaptic responses (latency, 6-106 ms) were evoked from sites both within and outside the dendritic field (Fig. 1D2, top right), and were abolished by TTX (bottom right); this shows that there was no contamination of the synaptic response map by direct responses. 
A
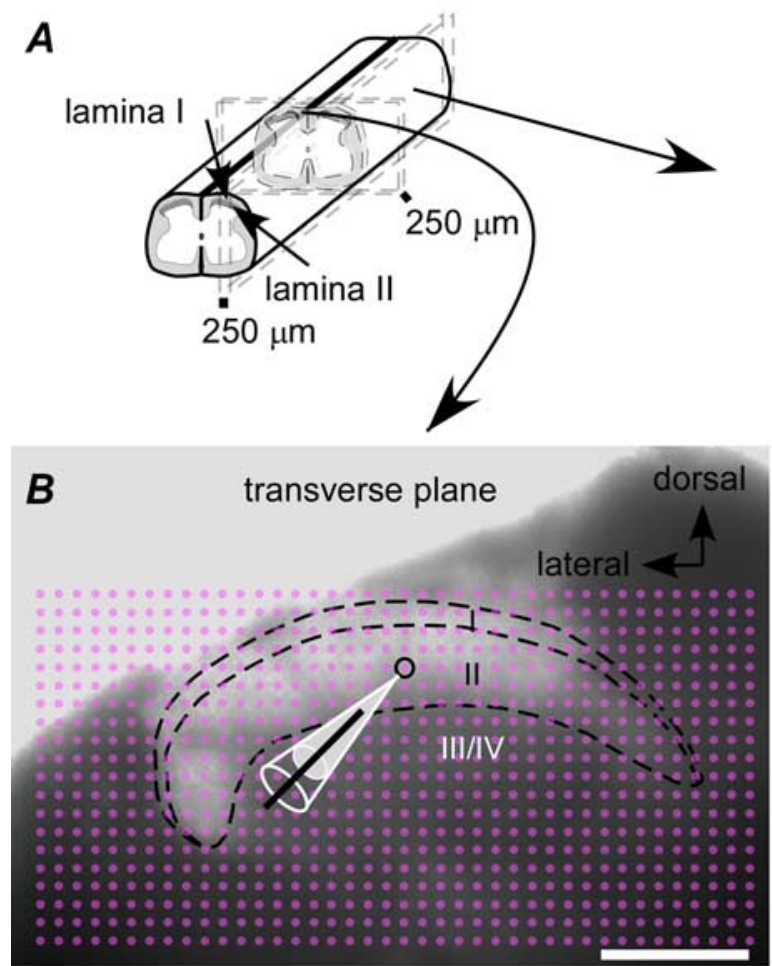

D2
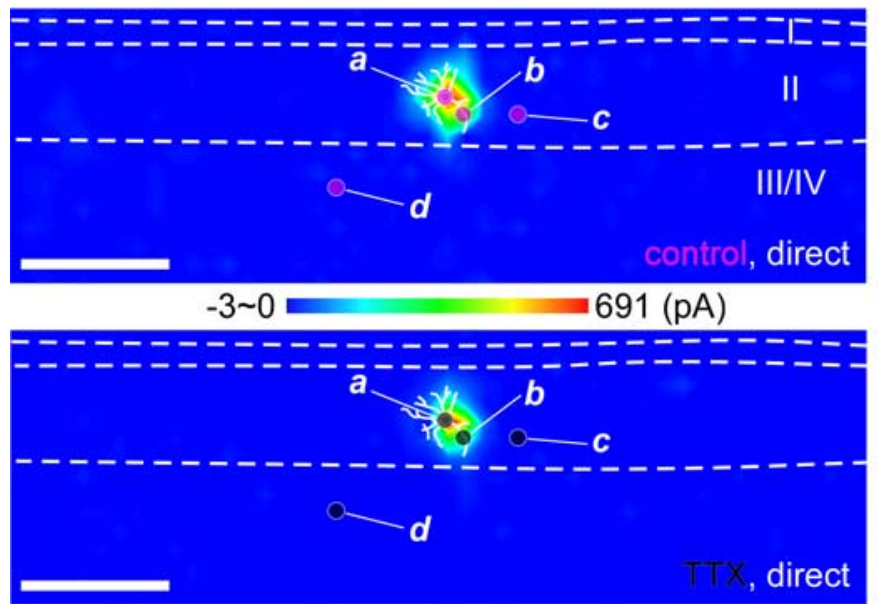
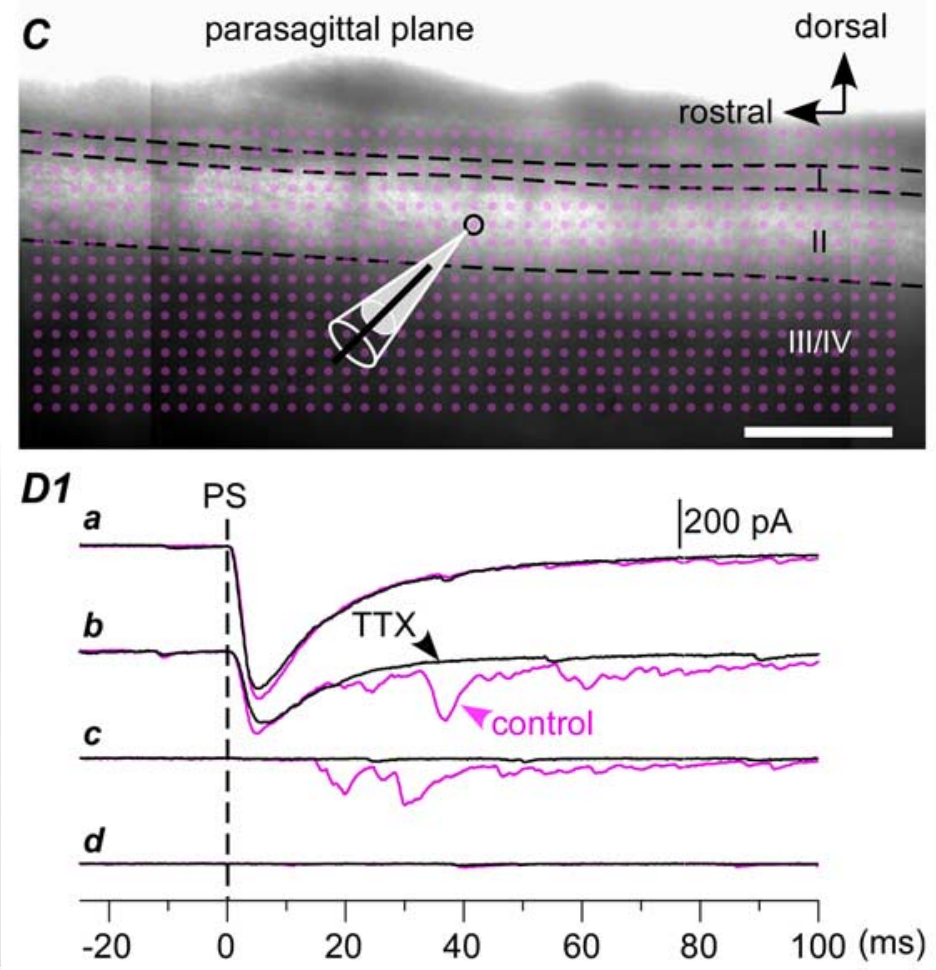

Figure 1. Illustration of photostimulation (PS) grids in transverse and parasagittal slices, and identification of direct versus synaptically evoked responses. Diagram ( $\boldsymbol{A}$ ) and IR-DIC images (B, $\boldsymbol{C})$ of transverse and parasagittal spinal cord slice preparations. The purple dots in $\boldsymbol{B}$ and $\boldsymbol{C}$ indicate the PS scanning grids used for mapping of sites that evoke synaptic responses (800 sites in a $20 \times$ 40 array for transverse, 768 sites in a $16 \times 48$ array for parasagittal, with $25 \mu \mathrm{m}$ spacing). The black circles in $\boldsymbol{B}$ and $\mathbf{C i n d i c a t e ~ t h e ~ p o s i t i o n ~ o f ~ t h e ~ s o m a ~ o f ~ t h e ~ r e c o r d e d ~ n e u r o n . ~ D 1 , ~ D 2 , ~ I d e n t i f i c a t i o n ~}$ of direct versus synaptic responses based on TTX sensitivity. D1 shows the responses evoked by photostimulation at the four sites $(a-d)$ indicated in the maps in $\mathbf{D 2}$, recorded in a radial cell in the presence (black traces) and absence (purple traces) of TTX. TTX blocked late ( $>6 \mathrm{~ms}$ delay) but not early responses. Direct and synaptic responses were therefore determined using response time windows of $0-5$ and $6-106 \mathrm{~ms}$, respectively (Kato et al., 2007). In D2, the top and bottom left maps show that direct responses were TTX-resistant, whereas the top and bottom right maps show that synaptic responses were blocked by TTX. Stimulation at the soma (site $a$ ) evoked a large direct response, whereas stimulation at the dendritic site $b$ evoked a mixture of direct and synaptic (EPSCS) responses, indicating the presence of a presynaptic partner at this site. Stimulation away from the dendritic sites evoked a purely synaptic response (site c) or no significant increase above spontaneous activity (site $d$ ), indicating the presence or absence of presynaptic partners at those respective sites. Maps were constructed from the sum of the peak amplitudes of the inward currents. Holding potential, $-70 \mathrm{mV}$. Scale bars, $200 \mu \mathrm{m}$.

Comparison between distribution of synaptic input zones revealed in transverse and parasagittal slices

Comparison of transverse and parasagittal maps of synaptic input zones of lamina II neurons showed that these input zones had a predominantly sagittal orientation, in that they had a much larger extent in the rostrocaudal than the mediolateral dimension (Fig. 2). This sagittal orientation was exhibited by both the excitatory (Fig. 2A-D,G) and inhibitory (Fig. 2E, F,I) synaptic input zones. In addition, the averaged excitatory input zone had a much more widespread rostrocaudal distribution than the averaged inhibitory input zone. Examples of individual excitatory input maps are shown for two lamina IIo vertical cells (Fig. $2 A, B)$, and soma-aligned averaged excitatory and inhibitory input maps are shown for all lamina II neurons [EPSC (Fig. 2C,D); IPSC (Fig. 2E, F)]. The rostrocaudal and mediolateral distributions are compared in plots of EPSC and IPSC amplitude versus 
A

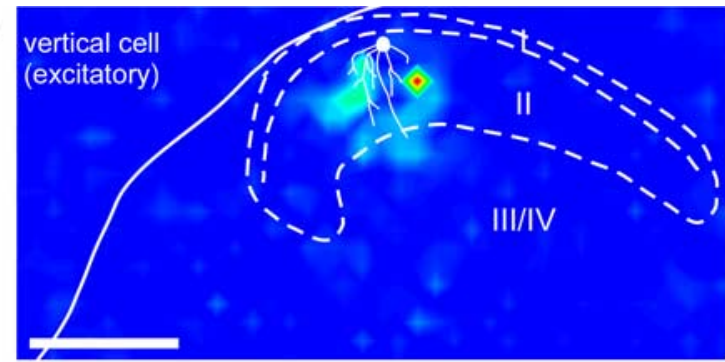

$-14 \sim 0=662(p A)$

C

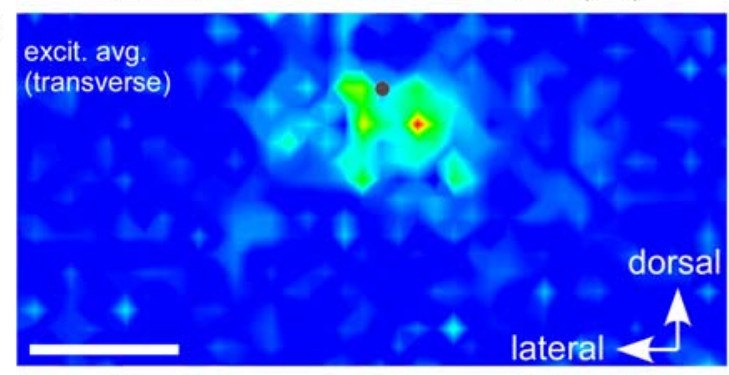

$-25 \sim 0=180(\mathrm{pA})$

E

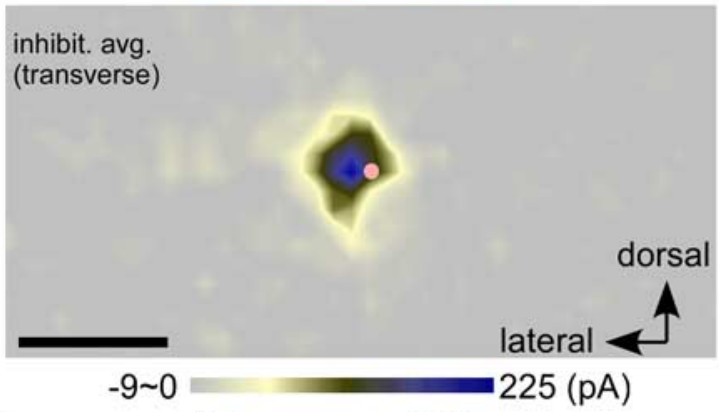

B

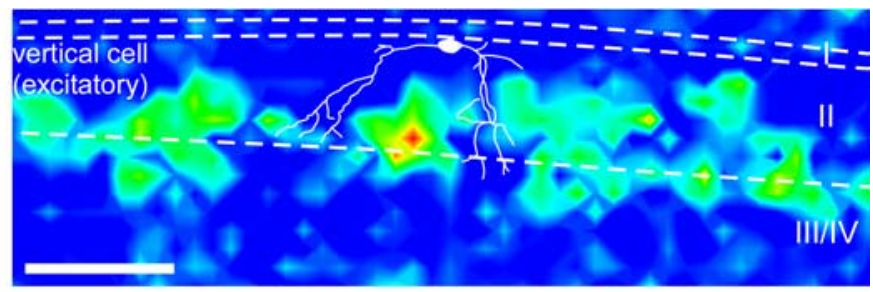

$-18 \sim 0=223(\mathrm{pA})$

D

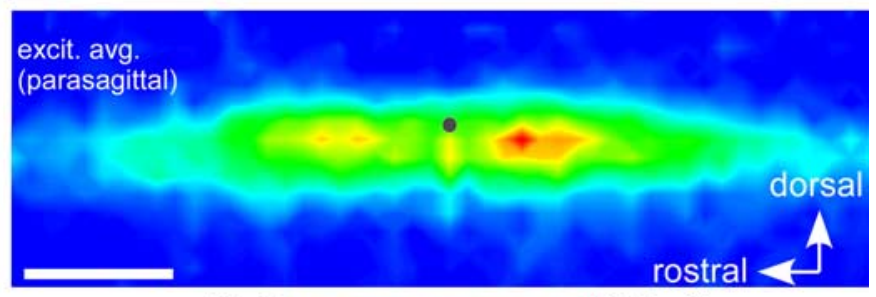

$-12 \sim 0=88(\mathrm{pA})$

$\boldsymbol{F}$

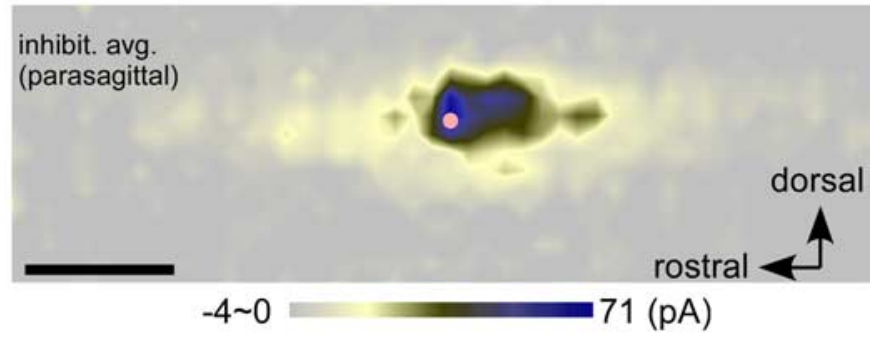

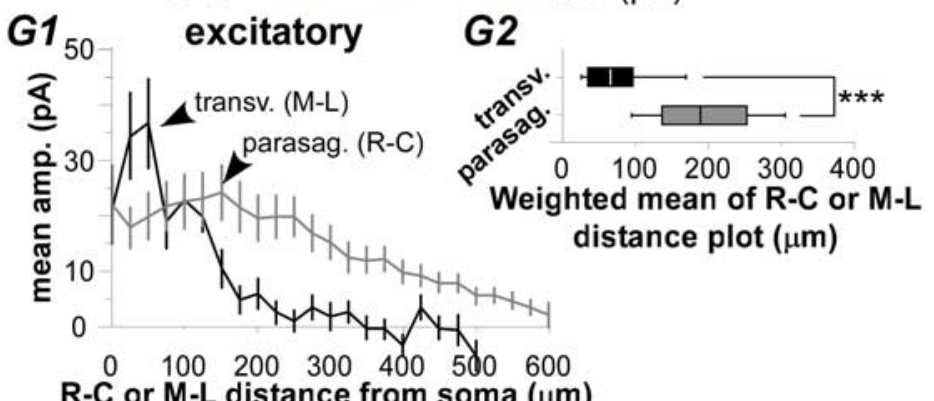
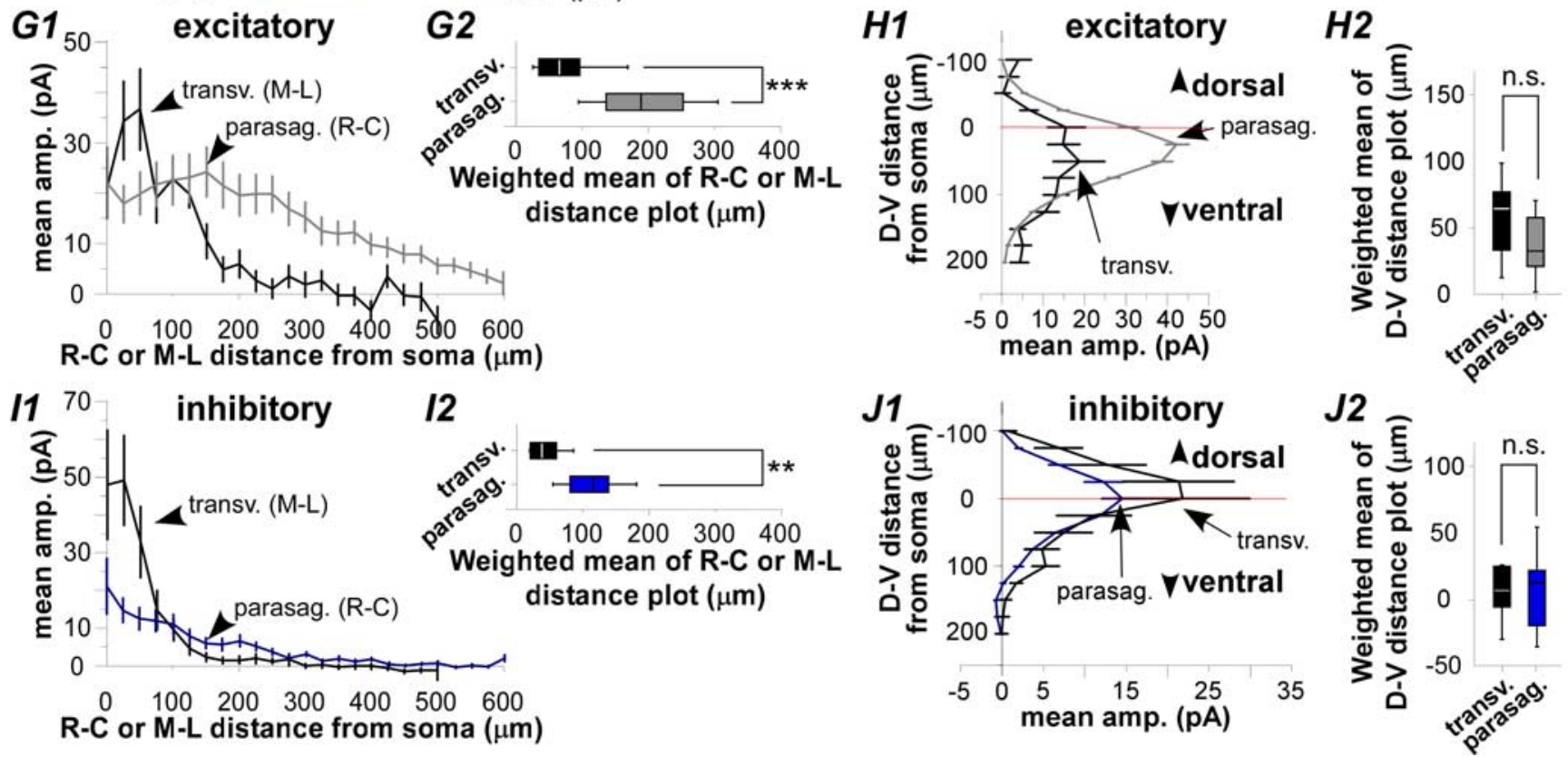

Figure 2. Comparison of excitatory and inhibitory synaptic input zones (sites that evoke EPSCs or IPSCs, respectively) of lamina II neurons mapped in the transverse and parasagittal planes. $\boldsymbol{A}, \boldsymbol{B}$, Examples of transverse $(\boldsymbol{A})$ and parasagittal $(\boldsymbol{B})$ maps of excitatory input zones for two lamina ll vertical cells. $\boldsymbol{C} \boldsymbol{F}$, Averaged transverse $(\boldsymbol{C})(n=8)$ and parasagittal $(\boldsymbol{D})(n=106)$ maps of excitatory input zones, and transverse $(\boldsymbol{E})(n=5)$ and parasagittal $(\boldsymbol{F})(n=19)$ maps of inhibitory input zones, for all lamina II neurons. For averaging, maps of individual neurons were aligned by soma location ( $\boldsymbol{C}-\boldsymbol{F}$, gray circles). $\mathbf{G 1}$, Plots of mean response amplitude versus mediolateral (black) and rostrocaudal (gray) distance from the soma as measured from averaged transverse and parasagittal maps of excitatory input zones, respectively. G2, Box plots of the mean distance of the input zone from the soma, as measured along the mediolateral and rostrocaudal axes (calculated as the weighted mean of the plots in G1). $\mathbf{H 1}, \mathbf{H 2}$, Same plots as G1, G2, but in the dorsoventral direction, obtained from both transverse (black) and parasagittal (gray) planes. I1, Plots of mean response amplitudes versus mediolateral (black) and rostrocaudal (navy) distance from the soma as measured from averaged transverse and parasagittal maps of inhibitory input zones, respectively. $\mathbf{2}$, Box plots of the mean distance of the input zone from the soma, as measured along the mediolateral and rostrocaudal axes (calculated as the weighted mean of the plots in I1). $\mathbf{J 1}, \mathbf{J 2}$, Same plots as $\mathbf{I 1}$ and $\boldsymbol{I} \mathbf{2}$ but in the dorsoventral direction, obtained from both transverse (black) and parasagittal (navy) planes. 
distance of the stimulus site from the soma (Fig. 2G1,I1). The weighted means of the plots in Figure 2, G1 and I1, were calculated as a measure of the center of the distribution relative to the soma (Fig. 2G2,I2). These values were significantly greater for the rostrocaudal than the mediolateral distribution (EPSC, $194 \pm$ 8.00 vs $78.5 \pm 20.2 \mu \mathrm{m}, p=0.0002$; IPSC, $111 \pm 11.1$ vs $41.6 \pm$ $12.2 \mu \mathrm{m}, p=0.006)$. The dorsoventral distributions were very similar when mapped in the transverse versus the parasagittal planes (Fig. $2 \mathrm{H1}, \mathrm{J1}$ ) [no significant difference in weighted means (Fig. $2 H 2, J 2), p=0.11$ and 0.88 , respectively]. The dorsoventral plots also show that the excitatory input zones arose mostly from sites whose dorsoventral level was ventral to that of the soma, whereas the inhibitory input zones were approximately centered dorsoventrally on the soma (Figs. 2H1,J1, 4E1).

\section{Cell type-specific patterns of intralaminar and translaminar excitatory synaptic inputs to lamina II neurons}

Figure 3 shows parasagittal maps of excitatory input zones for two individual lamina II neurons (Fig. $3 A 1, A 2$ ) and averaged population maps for each of the four major lamina II cell classes (Fig. $3 B-F$ ), in which individual maps were aligned dorsoventrally by the lamina II borders to illustrate the laminar distribution of the input zones (whereas individual maps were aligned dorsoventrally by soma location for the averaged maps in Fig. 2). The laminar-aligned population maps show the presence of both intralaminar inputs, from within lamina II, as well as translaminar inputs, from lamina III-IV. Inputs from lamina I were mostly absent. The central, radial, and islet cell populations received input almost entirely from within lamina II, including substantial input from both IIo and IIi (Fig. $3 B-D, H$ ). The vertical cell population received an additional input from lamina III-IV. The lamina III-IV input was primarily found in a subset of vertical cells, which were distinguished by the more ventral location of their somata. To illustrate this point, the vertical cells were subdivided into two groups based on soma location: those in the outermost part of lamina II ("IIo" cells) and those in the midportion of the lamina near the border between IIo and IIi ("IIm" cells) (Fig. $3 E, F$, respectively). Vertical cells were mostly absent from the inner one-half of lamina II, consistent with previous studies (Grudt and Perl, 2002; Yasaka et al., 2007). Similar to the nonvertical cells, the IIo vertical cells also received the majority of their input from within lamina II, but their averaged input zone was overall much larger, and extended further ventrally to include the lamina II-III border region (Fig. $3 E, H$ ). In contrast, the IIm vertical cells received substantial input from lamina III-IV, as well as from IIi, and almost no input from IIo. The lamina III-IV input to these cells represents the one major input from outside lamina II to any of the lamina II populations. A lamina III-IV input zone was found for 18 of 30 vertical cells (IIo vertical, 8 of 19; IIm vertical, 10 of 11), as well as 3 radial cells and 2 unclassified cells. Lamina I input zones were found for a small minority of neurons (one islet, two central, two vertical, two radial).

The differing patterns of intralaminar and translaminar inputs exhibited by the lamina II cell types can be understood as resulting from the presence of two distinct patterns in the dorsoventral distribution of inputs relative to the dorsoventral position of the soma of the neuron (Fig. 3H1). For all of the cell types, the excitatory input to individual neurons came predominantly from regions ventral to the position of the soma of the neuron, but this ventral displacement was much greater for the vertical cells than for the other cell types. Consequently, the vertical cells received a greater proportion of their input from the lamina or sublamina ventral to that of their own soma, compared with the other cell types. None of the lamina II cell types received the major portion of their input from regions dorsal to their own soma. This suggests that the direction of excitatory transmission within lamina II is predominantly upward (dorsal).

These two distinct dorsoventral patterns of input exhibited by the vertical and nonvertical cell populations parallel the difference between the two groups in the dorsoventral extent of their dendritic fields. All four of the cell types exhibit a ventral skewing in the shape of their dendritic fields, in that the ventral extent is greater than the dorsal extent (Grudt and Perl, 2002; Yasaka et al., 2007). However, the vertical cells have a far greater ventral dendritic extent than the other cell types. These patterns suggest that there is a relationship between the dorsoventral distribution of the dendritic field of a neuron and the dorsoventral distribution of its excitatory input zone. To examine this relationship, we mapped the sites that evoked direct responses (supplemental Fig. $1 A-D$, available at www.jneurosci.org as supplemental material) and used these direct response maps as a measure of the relative shape and distribution of the dendritic fields (because direct responses are only evoked from dendritic sites) (Fig. 1D2). Figure $3 I$ shows a plot of the weighted mean of the dorsoventral distribution of direct versus excitatory synaptic responses, for the lamina II cell groups ( $r=0.96 ; p=0.006$; Pearson's correlation) (supplemental Fig. 1I, available at www.jneurosci.org as supplemental material). The vertical cells showed a correspondingly greater ventral displacement of both their direct response sites (indicative of the dendritic field) and their excitatory synaptic input zone. The cell groups also exhibited differences in the rostrocaudal distribution of their excitatory synaptic input zones (Fig. 3G1,G2). However, the relationship between direct and synaptic response sites was much stronger for the dorsoventral distribution than the rostrocaudal distributions (Fig. 3I; supplemental Fig. $1 H$, I, available at www.jneurosci.org as supplemental material).

\section{Inhibitory synaptic input zones of lamina II neurons}

In our previous study of lamina II islet cells (Kato et al., 2007), we demonstrated that the inhibitory synaptic input zones were mostly restricted to the region immediately surrounding the soma of the neuron and had a much smaller rostrocaudal extent than the excitatory input zones. A similar proximal distribution of inhibitory input zones was found in the present study for all lamina II cell types (Figs. 2F, 4B) (averaged maps aligned by soma and laminar border, respectively). In most neurons (14 of $19 ; 74 \%)$, the site that evoked the maximum response was within $50 \mu \mathrm{m}$ rostrocaudally of the soma of the neuron. The inhibitory input zones had a much more restricted rostrocaudal distribution than the excitatory input zones (Fig. 4D1,D2). The weighted mean of the rostrocaudal plot was closer to the soma for the inhibitory than the excitatory input zones (Fig. 4D2) (202 \pm 9.52 vs $111 \pm 11.1 \mu \mathrm{m} ; p<0.0001)$, and the half-width was smaller $(138 \pm 32.5$ vs $340 \pm 24.3 \mu \mathrm{m} ; p=0.0008)$ (Table 1$)$.

In the dorsoventral dimension, the inhibitory and excitatory input zones were similar in size, as shown in plots of the distributions aligned by soma location (Fig. 4E1) (half-width, $59 \pm 4$ vs $71 \pm 3 \mu \mathrm{m}$, respectively; $p=0.13$ ) (Table 1). However, the excitatory zones for both vertical and nonvertical cells had a dorsoventral distribution that was skewed ventrally relative to the soma, whereas, as noted above, the inhibitory input zones did not show this ventral skewing. The averaged inhibitory input zone for the lamina II population as a whole extended to the dorsal and ventral borders of lamina II (Fig. $4 B, E 1, E 2)$. The inhibitory input 


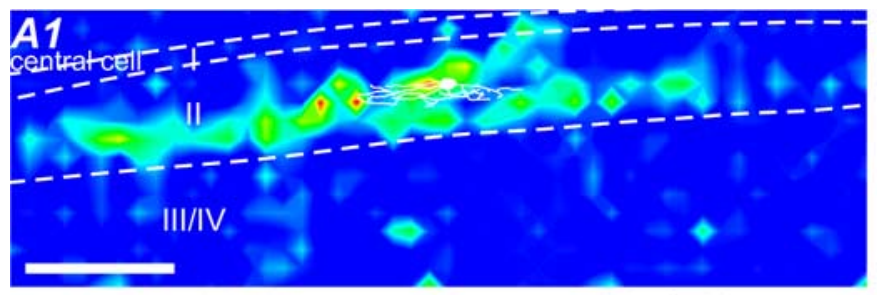

$-16 \sim 0=205(\mathrm{pA})$

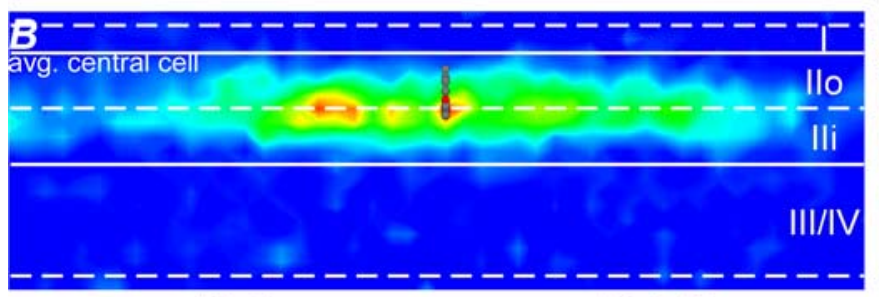

$-10 \sim 0=92(\mathrm{pA})$

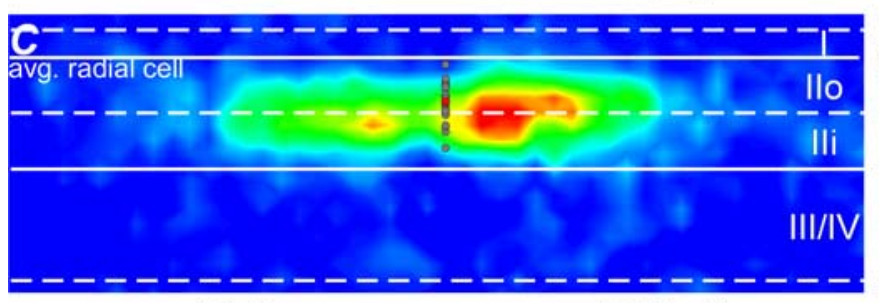

$-16 \sim 0=127(\mathrm{pA})$

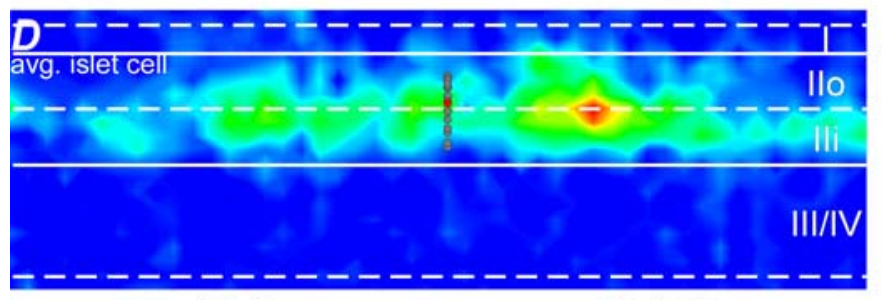

$-10 \sim 0=85(\mathrm{pA})$

\section{G1 50}

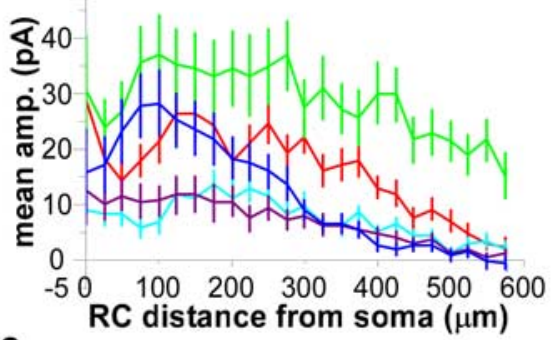

G2

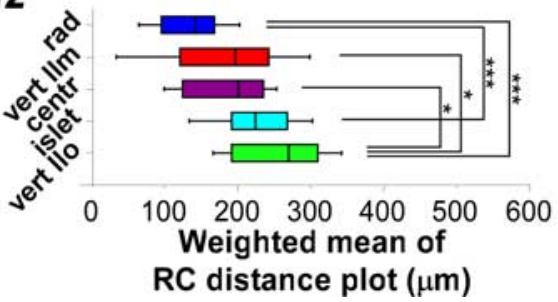

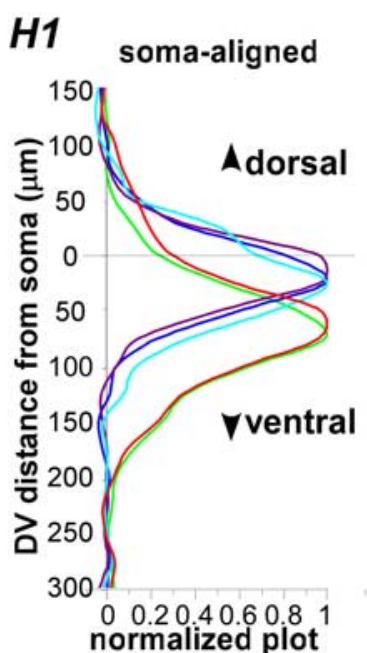

normalized plot

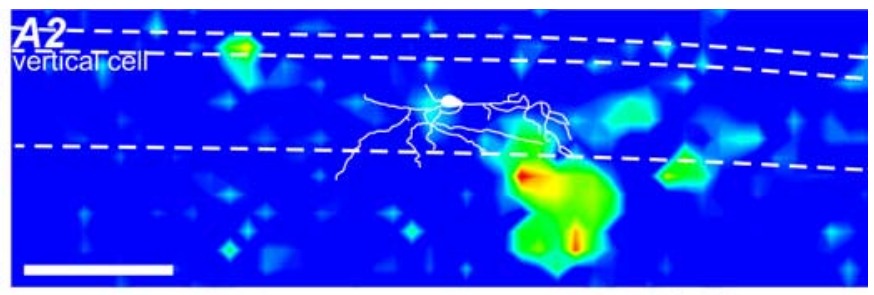

$-14 \sim 0=106(\mathrm{pA})$

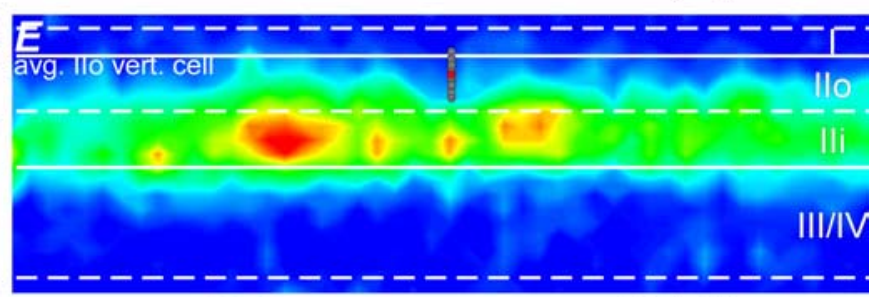

$-18 \sim 0=158(\mathrm{pA})$
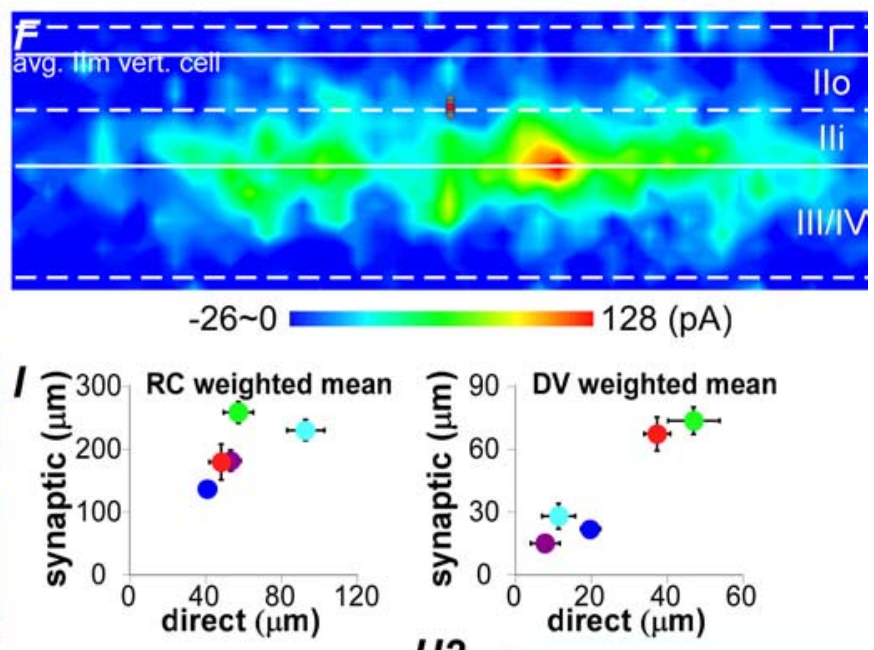

H2<smiles>C12C3C4C1C3C24</smiles>

家㔭

n.s.

laminar-aligned

जू०

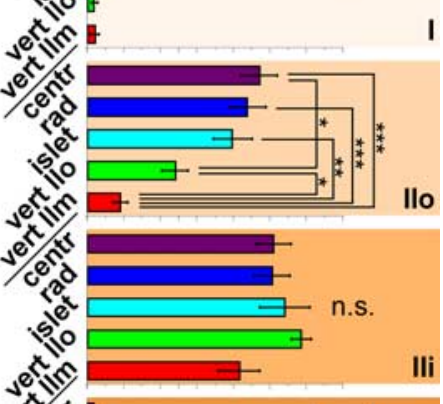
mean amp. (pA)
IIIIV

020406080100

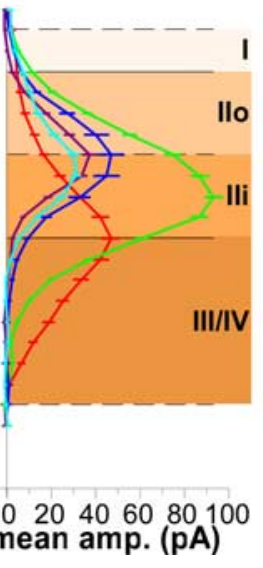

Figure 3. Comparison of excitatory input zones for different classes of lamina II neurons. $\mathbf{A 1}, \mathbf{A 2}$, Examples of excitatory synaptic input maps for a lamina ll central cell and vertical cell, respectively. $\boldsymbol{B}-\boldsymbol{F}$, Averaged excitatory synaptic input maps for lamina II central $(n=16)$, radial $(n=28)$, islet $(n=16)$, vertical Ilo $(n=19)$, and vertical IIm $(n=11)$ cells. For averaging, maps from individual neurons were aligned rostrocaudally by soma location and dorsoventrally by the borders of lamina II (white solid lines) (whereas averaged maps in Fig. 2 were aligned dorsoventrally by the soma). The gray and red circles mark the individual and averaged soma locations, respectively. G1, G2, Rostrocaudal distribution of excitatory input zones for the same five groups of lamina II neurons shown in the maps in $\boldsymbol{B}-\boldsymbol{F}$. G1, Plots of mean amplitude versus rostrocaudal distance from the soma. G2, Mean rostrocaudal distance of the input zone from the soma (calculated as the weighted mean of the plots in G1). $\boldsymbol{H}$, Dorsoventral and laminar distribution of excitatory input zones for the same five groups of lamina II neurons. H1, Plots of mean amplitude versus dorsoventral position, aligned by the soma (left) or the lamina II borders (right); amplitudes were normalized for the soma-aligned plot. $\mathbf{H 2}$, Bar graphs of the percentage of total inputs from each laminar region. I, Plots of the weighted mean of the rostrocaudal (left) and dorsoventral (right) distributions for direct versus synaptic excitatory responses. Correlation for dorsoventral, $r=0.96, p=0.0063 ;$ rostrocaudal, $r=$ $0.62, p=0.30$ ( $n=5$ groups). 


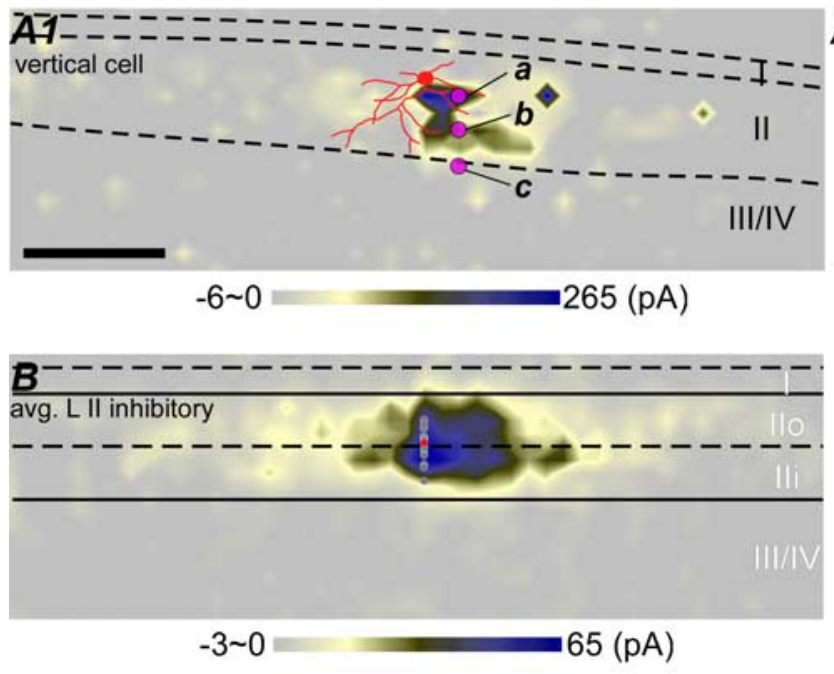

D1

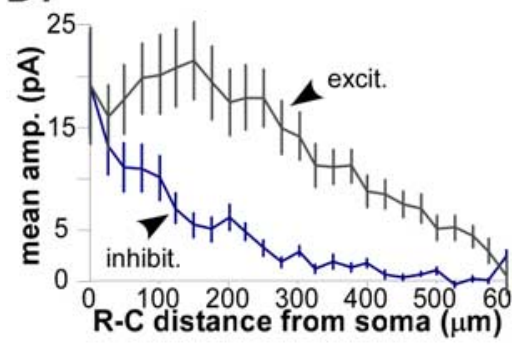

D2

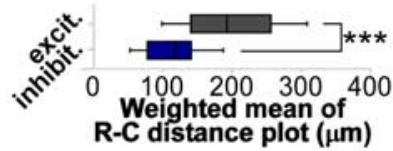

E1

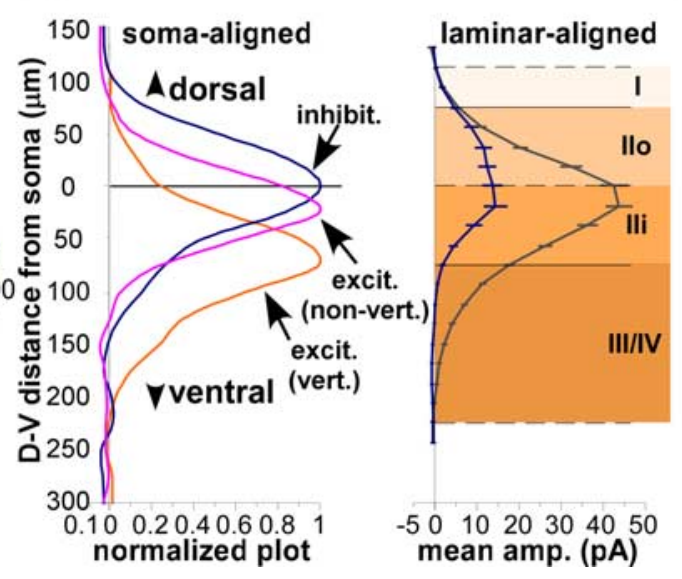

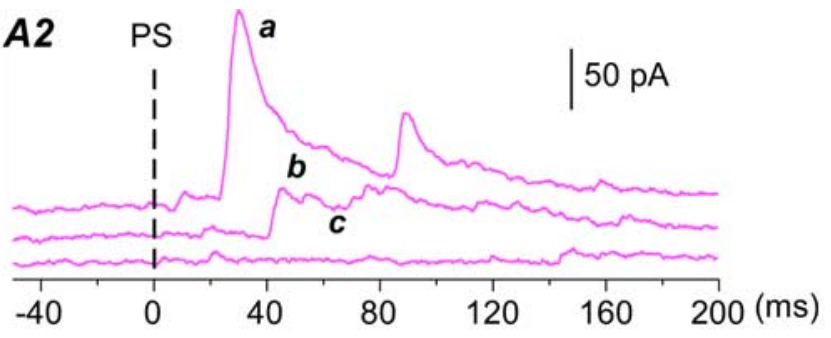

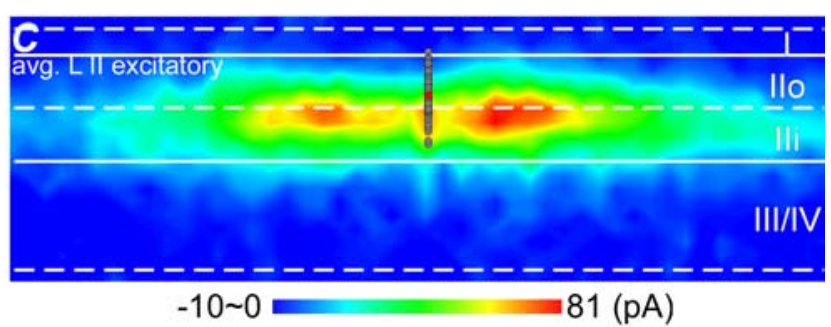

E2

Figure 4. Inhibitory synaptic zones of lamina II neurons and comparison with excitatory input zones. A1, $a$, Representative example of a map of the inhibitory input zone for a vertical cell, and $b$, responses evoked by stimulation at sites $a-c$. $B$, Averaged map of the inhibitory input zone for lamina II neurons $(n=19)$. Individual maps were aligned rostrocaudally by soma location and dorsoventrally by the borders of lamina II (black solid lines). C, Averaged map of the excitatory input zone for lamina ll neurons ( $n=106)$. $\boldsymbol{D}$, Rostrocaudal distribution of inhibitory input zones (navy line) and excitatory input zones (gray line) for lamina II neurons, as illustrated in plots of mean response amplitude versus rostrocaudal distance from the soma (D1) and box plots of the mean rostrocaudal distance of the input zone from the soma ( $\boldsymbol{D 2}$, calculated as the weighted mean of the plots in $\boldsymbol{D} 1)$. $\boldsymbol{E}$, Dorsoventral and laminar distribution of inhibitory and excitatory input zones for lamina II neurons. E1, Plots of mean amplitude versus dorsoventral position, aligned by the soma (left) or the lamina II borders (right); amplitudes were normalized for the soma-aligned plot. D2, Bar graphs of the percentage of total inputs from each laminar region.

Table 1. RC and DV half-widths of excitatory and inhibitory synaptic input zones of superficial spinal dorsal horn neurons

\begin{tabular}{lll}
\hline & RC half-width $(\mu \mathrm{m})$ & DV half-width $(\mu \mathrm{m})$ \\
\hline Lamina 1 excitatory $(n=13)$ & $511 \pm 95.8$ & $84.6 \pm 10.0$ \\
Lamina 1 inhibitory $(n=9)$ & $272 \pm 50.9$ & $91.7 \pm 11.8$ \\
Lamina 2 excitatory $(n=106)$ & $340 \pm 24.3$ & $70.8 \pm 3.08$ \\
Central $(n=16)$ & $402 \pm 59.8$ & $59.4 \pm 5.04$ \\
Radial $(n=28)$ & $202 \pm 30.9$ & $55.3 \pm 3.37$ \\
Islet $(n=16)$ & $348 \pm 64.2$ & $68.8 \pm 7.40$ \\
Ilo vertical $(n=19)$ & $420 \pm 70.2$ & $81.8 \pm 4.51$ \\
IIm vertical $(n=11)$ & $325 \pm 48.9$ & $95.8 \pm 13.8$ \\
Unclassified $(n=16)$ & $409 \pm 71.3$ & $73.4 \pm 11.5$ \\
Lamina 2 inhibitory $(n=19)$ & $138 \pm 32.5$ & $59.2 \pm 4.36$
\end{tabular}

Half-width, or width at half-peak, calculated as the total distance encompassing all sites that had greater than one-half of the maximum amplitude. Data are reported as mean \pm SEM. RC, Rostrocaudal; DV, dorsoventral.

zone extended into lamina I in one dorsally placed vertical cell, and into the outer part of lamina III in one ventrally located radial cell, but otherwise was confined to lamina II. Overall, there was very little excitatory or inhibitory input found to lamina II neurons from lamina I.

\section{Excitatory and inhibitory synaptic input zones of lamina} I neurons

The excitatory input zones of lamina I neurons were much larger overall than those of lamina II neurons in both their laminar and rostrocaudal distribution (Fig. $5 \mathrm{~A}, \mathrm{C}$ ). Unlike lamina II neurons, individual lamina I neurons commonly received substantial excitatory input from both inner and outer regions of lamina II, including the region of the lamina II-III border, as well as from lamina I. The larger rostrocaudal extent of the input zones of lamina I neurons was reflected in the respective values for the two populations for half-width $(512 \pm 96$ vs $340 \pm 24 \mu \mathrm{m} ; p=0.03$ ) (Table 1 ) and weighted mean of the rostrocaudal plots $(247 \pm 23$ vs $194 \pm 8.00 \mu \mathrm{m} ; p=0.03)$. The dorsoventral distribution of the input zones relative to the soma was broader and showed a greater ventral displacement than that of the lamina II neuron population as a whole (Fig. $5 F 1$, left). As a result, lamina I neurons received relatively greater input from lamina II than lamina I (Fig. 5F1, right; F2), and so exhibited a predominantly translaminar rather than intralaminar pattern of excitatory synaptic input. As with the lamina II neurons, the lamina I neurons exhib- 


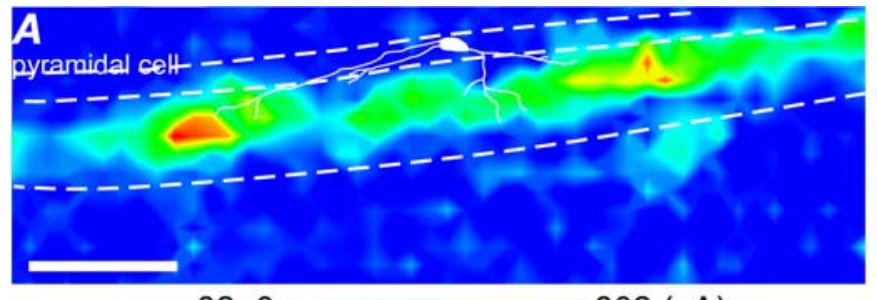

$-82 \sim 0=802(\mathrm{pA})$

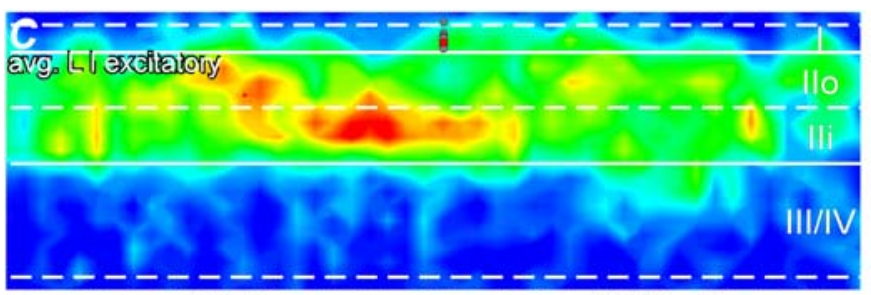

$-14 \sim 0=135(\mathrm{pA})$

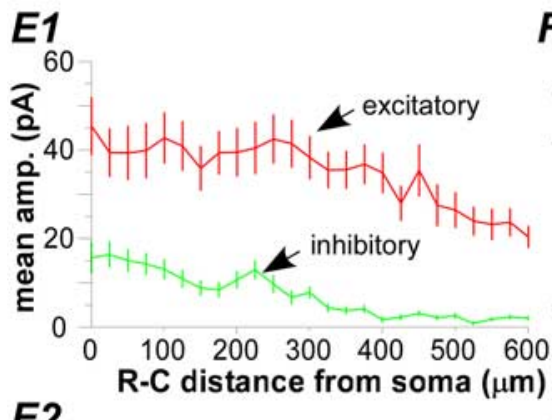

E2

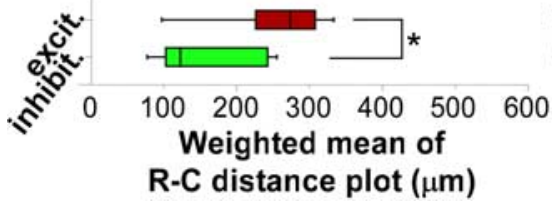

$F 1_{150}$

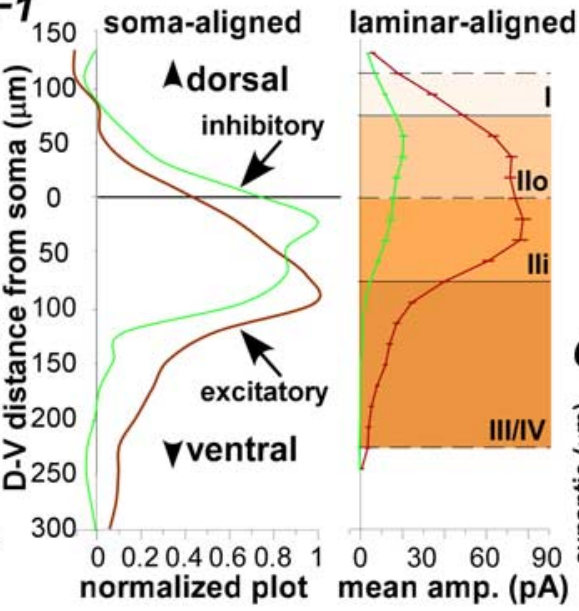

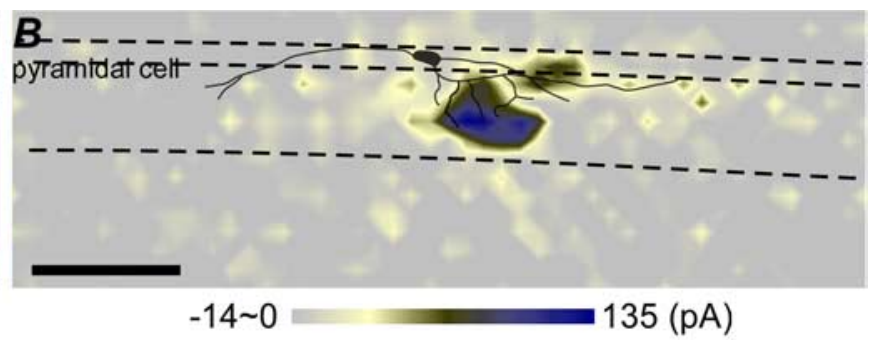

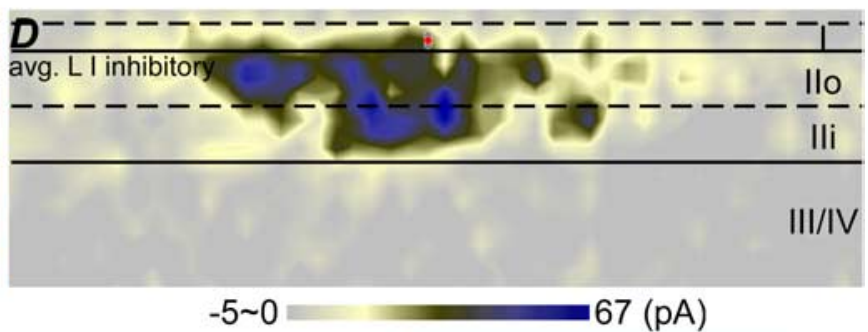

F2

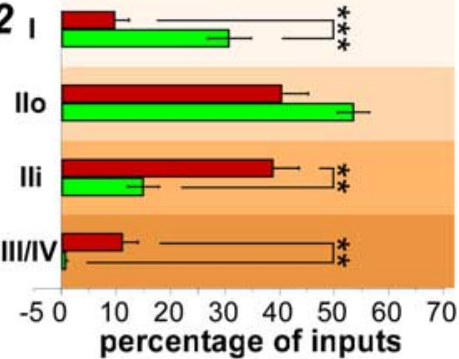

G RC weighted mean DV weighted mean

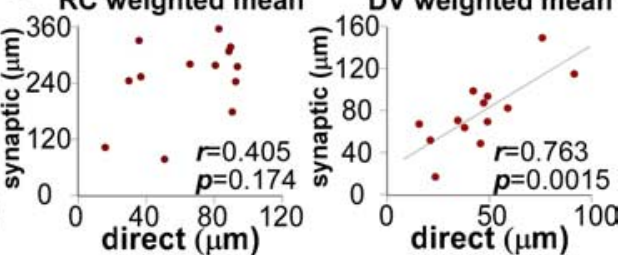

Figure 5. Excitatory and inhibitory input zones of lamina I neurons. Examples of individual maps $(\boldsymbol{A}, \boldsymbol{B})$ and averaged maps $(\boldsymbol{C}, n=13 ; \boldsymbol{D}, n=9)$ of excitatory and inhibitory input zones, respectively, for lamina I neurons. For averaging, individual maps were aligned rostrocaudally by soma location and dorsoventrally by lamina II borders (solid lines). $\boldsymbol{E}$, Rostrocaudal distribution of excitatory and inhibitory input zones for lamina I neurons, as illustrated in plots of mean response amplitude versus rostrocaudal distance from the soma (E1) and box plots of the mean rostrocaudal distance of the input zone from the soma (E2) (calculated as the weighted mean of the plots in $\boldsymbol{E} 1$ ). $\boldsymbol{F}$, Dorsoventral and laminar distribution of excitatory and inhibitory input zones for lamina I neurons. F1, Plots of mean amplitude versus dorsoventral position, aligned by the soma (left) or the lamina ll borders (right); amplitudes were normalized for the soma-aligned plot. F2, Bar graphs of the percentage of total inputs from each laminar region. $\mathbf{G}$, Plots of the weighted mean of the rostrocaudal (left) and dorsoventral (right) distributions for direct versus synaptic excitatory responses. $r$, Pearson's correlation coefficient.

ited a significant correlation between the dorsoventral distributions of their synaptic and direct responses (Fig. 5G). The correlation for the rostrocaudal distributions was not significant. These correlations (Fig. 5G; supplemental Fig. $1 H$, I, available at www.jneurosci.org as supplemental material) indicate that, within the superficial dorsal horn, the location of excitatory presynaptic neurons is strongly influenced by the dendritic vector of the postsynaptic neuron in the dorsoventral, but not the rostrocaudal axis.

The inhibitory input zones of lamina I neurons had a more dorsal distribution than the excitatory input zones (Fig. $5 B, D, F$ ). A greater percentage of the total inhibitory input arose from within lamina I as well as lamina IIo, compared with the excitatory input.

The inhibitory input zones were more restricted rostrocaudally than the excitatory input zones (Fig. 4E1,E2), although they were more widespread, both rostrocaudally and dorsoventrally, than the inhibitory input zones of lamina II neurons. Unlike the inhibitory input zones of lamina II neurons, the inhibitory input zones of lamina I neurons were shifted ventrally relative the soma (Fig. 5E1). As a result of this ventral displacement, lamina I neurons received a greater percentage of their inhibitory inputs from lamina II than lamina I, especially lamina IIo, and so exhibited a predominantly translaminar pattern of inhibitory synaptic input (Fig. 6).

\section{Discussion}

The present results reveal a three-dimensional spatial organization in the pattern of intrinsic connectivity within the superficial dorsal horn. Excitatory inputs to individual neurons arose from a much larger rostrocaudal extent than inhibitory inputs. The excitatory interneuronal connections exhibited a predominantly sagittal orientation and support a predominantly upward (dorsal) flow of excitatory transmission within the superficial laminae. The results reveal a pattern of translaminar connectivity, including an input from the deep dorsal horn, subserved by a 
excitatory wiring

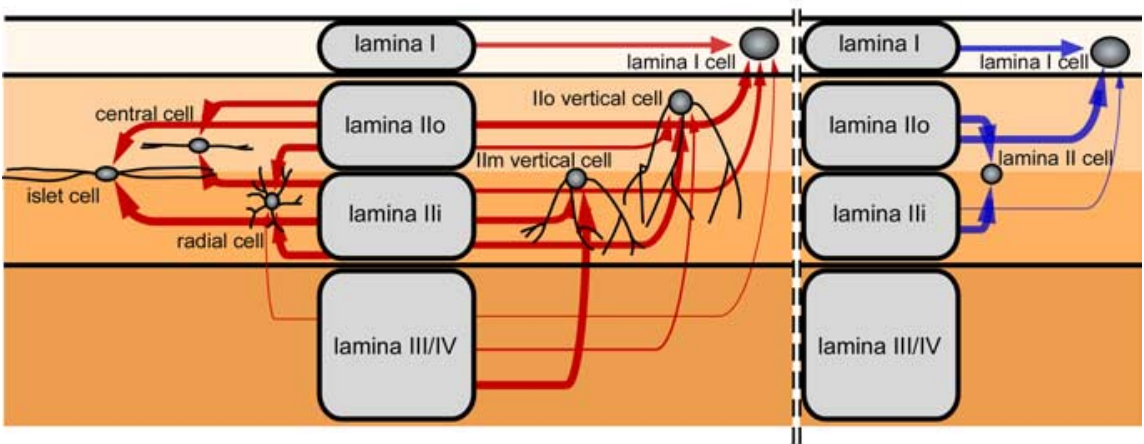

Figure 6. Proposed intralaminar and translaminar patterns of connectivity in the wiring of local excitatory and inhibitory synaptic inputs to superficial dorsal horn neurons.

specific subset of lamina II neurons, which could potentially support the polysynaptic transmission of low-threshold (tactile) inputs to nociceptive neurons. These spatial patterns of intrinsic connectivity thus have implications for processing of information related to both somatotopy and sensory modality.

\section{Somatotopy}

The neuropil of the dorsal horn, and especially the superficial laminae, exhibits a sagittal orientation that is apparent in both the primary afferent terminal fields as well as the dendritic fields of dorsal horn neurons (Szentagothai, 1964; Light and Perl, 1979; Sugiura et al., 1986; Grudt and Perl, 2002; Yasaka et al., 2007). The present results show that this predominantly sagittal orientation, with a highly restricted mediolateral dimension, is also exhibited in the functional connectivity of local excitatory projections within lamina II. This pattern of local connectivity might serve to both preserve and enhance the primary afferent somatotopy, by reinforcing the most somatotopically appropriate inputs. Brown et al. (1991) calculated that the dimensions of primary afferent terminations and dorsal horn dendritic fields predicted larger receptive field sizes than what is observed and concluded that some additional processing or filtering of the monosynaptic primary afferent input must occur to account for the observed somatotopic precision. One way such filtering could occur would be if the postsynaptic neurons required some degree of spatial summation of inputs to reach threshold, such as from convergence of inputs from multiple primary afferents (Pinto et al., 2008) or other dorsal horn neurons with overlapping central terminal fields. In this case, the spatial selectivity of the excitatory interneuronal connections might be one factor that contributes to a sharpening of the somatotopic precision beyond what would be generated by monosynaptic primary afferent connections alone.

\section{Inhibitory input zones}

Inhibitory inputs to lamina II neurons arose from a relatively restricted area around the soma in both the rostrocaudal and mediolateral dimensions, which suggests that the widespread inhibitory receptive fields found in dorsal horn neurons are mediated by longranging primary afferent or descending inputs, rather than long interneuronal connections (cf. Kato et al., 2004). The inhibitory synaptic input zones were much more restricted in their rostrocaudal dimension than the excitatory input zones [as found previously for islet cells (Kato et al., 2007)]. This could be explained if inhibitory interneurons had shorter axons than excitatory interneurons. However, there is no evidence for such a difference, and it has been found that islet cells, which are inhibitory, can have dense axonal arboriza- tions within lamina II at relatively long distances from their soma (Grudt and Perl, 2002; Maxwell et al., 2007). Possibly such long-range inhibitory inputs primarily target dendrites of neurons from other laminae. This possibility would be consistent with our finding that lamina I neurons do receive substantial inhibitory input from lamina II, and this input arises over longer rostrocaudal distances than the inhibitory input to lamina II neurons. This possibility would also be consistent with the finding of Santos et al. (2007) that lamina II neurons more commonly make inhibitory projections to neurons in lamina I than in lamina II. Such inhibitory projections from lamina II neurons to lamina I neurons presumably are made on dendrites that extend ventrally into lamina II, because the currently available evidence indicates that the axonal arborizations of islet cells as well as other lamina II inhibitory interneurons do not enter lamina I (Grudt and Perl, 2002; Maxwell et al., 2007).

Another factor that could partly account for a difference in the size of excitatory and inhibitory input zones would be if there were a difference in the somadendritic distribution of postsynaptic sites, such that excitatory inputs had a relatively greater distribution on distal dendrites than inhibitory inputs (cf. Kato et al., 2007). In the absence of a difference in the length of excitatory and inhibitory axons, such a difference in postsynaptic sites would itself be expected to result in larger excitatory input zones.

\section{Laminar organization and sensory modality}

Lamina I-II neurons exhibited cell type-specific patterns in the laminar distribution of their excitatory inputs that were related to their dorsoventral dendritic expanse. All cell types received excitatory input predominantly from positions ventral to that of their soma, but in lamina I neurons and lamina II vertical cells this ventral displacement of the excitatory input zone was greater than in the other lamina II cell types. As a result, these cells received a relatively greater proportion of their excitatory inputs from the lamina or sublamina ventral to that of their own soma ("translaminar" input) compared with the other lamina II cell types. None of the cell types received the major portion of its input from positions dorsal to that of its own soma. This implies that excitatory information flow within the superficial dorsal horn is predominantly upward (dorsal). In contrast, inhibitory input zones of lamina II neurons did not show this ventral skewing, but rather were centered dorsoventrally on the soma. The outer part of lamina II contains nociceptive (as well as thermoreceptive) neurons, whereas the inner part of lamina II contains primarily mechanoreceptive neurons (Light, 1992; Light and Willcockson, 1999). Therefore, the upward flow of excitatory intrinsic connectivity could potentially support a polysynaptic transmission of mechanoreceptive input to nociceptive neurons. By virtue of its polysynaptic nature, it would be expected that such indirect input would normally be weaker than direct monosynaptic input, but also show greater modifiability as a result of changes in synaptic strength that can occur during conditions of altered pain sensitivity.

In addition to a greater ventral displacement of the excitatory input zone than the other lamina II cell types, vertical cells exhibit a parallel difference in the dorsoventral dimensions of their dendritic fields, in that their dendrites extend much further ventrally from the soma than those of the other lamina II cell types (Grudt 
and Perl, 2002; Yasaka et al., 2007). The dendritic fields of all the lamina II cell types are skewed ventrally relative to the soma, but this ventral skewing is much greater in the vertical cells. There thus appears to be a relationship among the lamina II cell classes between the dorsoventral distribution of the dendrites of a neuron and the dorsoventral distribution of the neurons from which it receives local excitatory projections. One way such a relationship could arise would be if the axons that subserve excitatory connections between lamina II neurons preferentially targeted postsynaptic dendritic sites that were at a similar dorsoventral level to their own soma. In contrast to the excitatory input zones, the inhibitory input zones for lamina II neurons did not show a ventral skewing that mirrored the dendritic distribution, but instead were centered dorsoventrally on the soma. This distribution could also be explained if inhibitory interneurons targeted postsynaptic sites at a similar dorsoventral level as their own soma, but tended to target somatic rather than dendritic sites. Such a preferential targeting of somatic versus dendritic sites by inhibitory and excitatory interneurons would be generally consistent with the findings of a number of previous studies in various central neurons, including our previous study on lamina II islet cells, on the differential somatodendritic distribution of inhibitory versus excitatory synapses [discussed by Kato et al. (2007)].

A critical finding of the present study was the presence of a previously unknown lamina III-IV input to the superficial dorsal horn. This input was mostly confined to a specific subgroup of lamina II neurons, the vertical cells, particularly the deepest vertical cells located near the IIo/IIi border ("IIm" vertical cells). Lamina III-IV receives projections from low-threshold mechanoreceptive A- $\beta$ fiber primary afferents (Brown et al., 1977; Woolf, 1987), and electrophysiological studies have demonstrated monosynaptic A- $\beta$ input to neurons in this region, including neurons just ventral to the lamina II border (Torsney and MacDermott, 2006). Therefore, the input from lamina III-IV found in the present study represents a potential route for polysynaptic low-threshold mechanoreceptive A- $\beta$ input to reach neurons in the superficial dorsal horn neuron. Such polysynaptic A- $\beta$ input has been demonstrated previously for neurons in both lamina I (Torsney and MacDermott, 2006) and II (Baba et al., 2003). The present study found major input to lamina I neurons from lamina II, but only relatively minor inputs from lamina III-IV; however, such inputs could be relayed to lamina I neurons via the known axonal projections of vertical cells to lamina I neurons (Lu and Perl, 2005). The percentage of superficial dorsal horn neurons that receive this polysynaptic $\mathrm{A}-\beta$ input is normally relatively low but can be increased by inhibitory transmitter blockade (Torsney and MacDermott, 2006) and also increases in animal models of inflammatory or neuropathic pain hypersensitivity (Baba et al., 1999; Nakatsuka et al., 1999; Okamoto et al., 2001; Kohno et al., 2003). The increase in A- $\beta$ input to superficial dorsal horn neurons could contribute to the mechanical hypersensitivity that characterizes such models, as well as the A- $\beta$-mediated mechanical allodynia (pain from a normally innocuous stimulus) that can accompany clinically occurring states of pain hypersensitivity in humans.

\section{References}

Baba H, Doubell TP, Woolf CJ (1999) Peripheral inflammation facilitates A- $\beta$ fiber-mediated synaptic input to the substantia gelatinosa of the adult rat spinal cord. J Neurosci 19:859-867.

Baba H, Ji RR, Kohno T, Moore KA, Ataka T, Wakai A, Okamoto M, Woolf CJ (2003) Removal of GABAergic inhibition facilitates polysynaptic A fibermediated excitatory transmission to the superficial spinal dorsal horn. Mol Cell Neurosci 24:818-830.

Bentley GN, Gent JP (1994) Electrophysiological properties of substantia gelatinosa neurones in a novel adult spinal slice preparation. J Neurosci Methods 53:157-162.

Braz JM, Nassar MA, Wood JN, Basbaum AI (2005) Parallel "pain” pathways arise from subpopulations of primary afferent nociceptor. Neuron 47:787-793.

Brown AG, Rose PK, Snow PJ (1977) Morphology of hair follicle afferent fibre collaterals in the cat spinal cord. J Physiol 272:770-797.

Brown PB, Gladfelter WE, Culberson JC, Covalt-Dunning D, Sonty RV, Pubols LM, Millecchia RJ (1991) Somatotopic organization of single primary afferent axon projections to cat spinal cord dorsal horn. J Neurosci 11:298-309.

Callaway EM, Katz LC (1993) Photostimulation using caged glutamate reveals functional circuitry in living brain slices. Proc Natl Acad Sci U S A 90:7661-7665.

Callaway EM, Yuste R (2002) Stimulating neurons with light. Curr Opin Neurobiol 12:587-592.

Dantzker JL, Callaway EM (2000) Laminar sources of synaptic input to cortical inhibitory interneurons and pyramidal neurons. Nat Neurosci 3:701-707.

Eckert WA 3rd, McNaughton KK, Light AR (2003) Morphology and axonal arborization of rat spinal inner lamina II neurons hyperpolarized by $\mu$-opioid-selective agonists. J Comp Neurol 458:240-256.

Grudt TJ, Perl ER (2002) Correlations between neuronal morphology and electrophysiological features in the rodent superficial dorsal horn. J Physiol 540:189-207.

Kato G, Furue H, Katafuchi T, Yasaka T, Iwamoto Y, Yoshimura M (2004) Electrophysiological mapping of the nociceptive inputs to the substantia gelatinosa in rat horizontal spinal cord slices. J Physiol 560:303-315.

Kato G, Kawasaki Y, Ji RR, Strassman AM (2007) Differential wiring of local excitatory and inhibitory synaptic inputs to islet cells in rat spinal lamina II demonstrated by laser scanning photostimulation. J Physiol 580:815-833.

Katz LC, Dalva MB (1994) Scanning laser photostimulation: a new approach for analyzing brain circuits. J Neurosci Methods 54:205-218.

Kohno T, Moore KA, Baba H, Woolf CJ (2003) Peripheral nerve injury alters excitatory synaptic transmission in lamina II of the rat dorsal horn. J Physiol 548:131-138.

Light AR (1992) The initial processing of pain and its descending control: spinal and trigeminal systems. Basel: Karger.

Light AR, Perl ER (1979) Spinal termination of functionally identified primary afferent neurons with slowly conducting myelinated fibers. J Comp Neurol 186:133-150.

Light AR, Willcockson HH (1999) Spinal laminae I-II neurons in rat recorded in vivo in whole cell, tight seal configuration: properties and opioid responses. J Neurophysiol 82:3316-3326.

Lu Y, Perl ER (2003) A specific inhibitory pathway between substantia gelatinosa neurons receiving direct C-fiber input. J Neurosci 23:8752-8758.

Lu Y, Perl ER (2005) Modular organization of excitatory circuits between neurons of the spinal superficial dorsal horn (laminae I and II). J Neurosci 25:3900-3907.

Maxwell DJ, Belle MD, Cheunsuang O, Stewart A, Morris R (2007) Morphology of inhibitory and excitatory interneurons in superficial laminae of the rat dorsal horn. J Physiol 584:521-533.

Nakatsuka T, Park JS, Kumamoto E, Tamaki T, Yoshimura M (1999) Plastic changes in sensory inputs to rat substantia gelatinosa neurons following peripheral inflammation. Pain 82:39-47.

Okamoto M, Baba H, Goldstein PA, Higashi H, Shimoji K, Yoshimura M (2001) Functional reorganization of sensory pathways in the rat spinal dorsal horn following peripheral nerve injury. J Physiol 532:241-250.

Pinto V, Szûcs P, Derkach VA, Safronov BV (2008) Monosynaptic convergence of $\mathrm{C}$ - and $\mathrm{A} \delta$-afferent fibres from different segmental dorsal roots on to single substantia gelatinosa neurones in the rat spinal cord. J Physiol 586:4165-4177.

Santos SF, Rebelo S, Derkach VA, Safronov BV (2007) Excitatory interneurons dominate sensory processing in the spinal substantia gelatinosa of rat. J Physiol 581:241-254.

Sawatari A, Callaway EM (2000) Diversity and cell type specificity of local excitatory connections to neurons in layer $3 \mathrm{~B}$ of monkey primary visual cortex. Neuron 25:459-471.

Sugiura Y, Lee CL, Perl ER (1986) Central projections of identified, unmyelinated (C) afferent fibers innervating mammalian skin. Science 234:358-361. 
Szentagothai J (1964) Neuronal and synaptic arrangement in the substantia gelatinosa Rolandi. J Comp Neurol 122:219-239.

Todd AJ, McKenzie J (1989) GABA-immunoreactive neurons in the dorsal horn of the rat spinal cord. Neuroscience 31:799-806.

Todd AJ, Sullivan AC (1990) Light microscope study of the coexistence of GABA-like and glycine-like immunoreactivities in the spinal cord of the rat. J Comp Neurol 296:496-505.

Torsney C, MacDermott AB (2006) Disinhibition opens the gate to pathological pain signaling in superficial neurokinin 1 receptor-expressing neurons in rat spinal cord. J Neurosci 26:1833-1843.

Willis WD, Coggeshall RE (2004) Sensory mechanisms of the spinal cord, Ed 3. New York: Kluwer Academic/Plenum Publishers.

Woolf CJ (1987) Central terminations of cutaneous mechanoreceptive afferents in the rat lumbar cord. J Comp Neurol 251:105-119.
Yasaka T, Kato G, Furue H, Rashid MH, Sonohata M, Tamae A, Murata Y, Masuko S, Yoshimura M (2007) Cell-type-specific excitatory and inhibitory circuits involving primary afferents in the substantia gelatinosa of the rat spinal dorsal horn in vitro. J Physiol 581:603-618.

Yoshimura M, Jessell TM (1989) Primary afferent-evoked synaptic responses and slow potential generation in rat substantia gelatinosa neurons in vitro. J Neurophysiol 62:96-108.

Yoshimura M, Nishi S (1992) Excitatory amino acid receptors involved in primary afferent-evoked polysynaptic EPSPs of substantia gelatinosa neurons in the adult rat spinal cord slice. Neurosci Lett 143:131-134.

Yoshimura M, Nishi S (1995) Primary afferent-evoked glycine- and GABAmediated IPSPs in substantia gelatinosa neurones in the rat spinal cord in vitro. J Physiol 482:29-38. 